\title{
Comparison of the Effect of Anemia on In-Hospital Mortality in Patients With Versus Without Preserved Left Ventricular Ejection Fraction
}

\author{
Adriana Lopes Latado, $\mathrm{PhD}^{\mathrm{a}, *}$, Luiz Carlos Santana Passos, PhD ${ }^{\mathrm{a}}$, Eduardo S. Darzé, MD ${ }^{\mathrm{b}}$, \\ and Antônio Alberto Lopes, $\mathrm{PhD}^{\mathrm{a}}$
}

\begin{abstract}
Patients with symptomatic heart failure (HF) frequently have preserved left ventricular (LV) ejection fractions (LVEFs). Although anemia is a common finding in this patient population, its prognostic role has not been well studied. This study's aim was to assess if the LVEF interferes in the association between anemia and in-hospital mortality in patients with severe HF. Consecutive patients admitted to an intensive care unit with decompensated chronic HF were prospectively enrolled. The diagnosis of HF was based on clinical criteria. Patients with LVEFs $\geq 45 \%$ (on echocardiography) were diagnosed as having preserved LVEFs. Multivariate analysis was performed to test the independent association between anemia and in-hospital mortality and to evaluate an interaction between anemia and systolic function. In all, 303 patients were recruited (mean age $69 \pm 13$ years; $45.5 \%$ women). Preserved LVEFs were present in $34 \%$ of the population. The prevalence of anemia in this group was $58 \%$, compared with $43 \%$ in the group with systolic dysfunction $(\mathrm{p}=0.01)$. Dilated left ventricles, left bundle branch blocks, and valvular dysfunction were significantly more frequent in patients with systolic heart failure. In-hospital mortality was similar in the groups with preserved LVEFs and systolic dysfunction $(p=0.71)$. On multivariate analysis, anemia was independently associated with in-hospital mortality (odds ratio $2.7,95 \%$ confidence interval 1.43 to $5.04, p=0.002$ ). There was no interaction between anemia and systolic function ( $\mathrm{p}=\mathbf{0 . 0 8}$ for interaction). In conclusion, anemia was an independent predictor of in-hospital mortality in symptomatic patients with severe HF, regardless of whether the patients had preserved or impaired LV systolic function. () 2006 Elsevier Inc. All rights reserved. (Am J Cardiol 2006;98:1631-1634)
\end{abstract}

The prognostic role of anemia in patients with heart failure (HF) and preserved left ventricular (LV) ejection fractions (LVEFs) was the subject of 3 recent studies. Brucks et $\mathrm{al}^{1}$ demonstrated a high prevalence of anemia $(42 \%)$ in patients with HF and preserved LVEFs. In that study, anemia was associated with a nonsignificant trend toward worse survival. More recently, in 2 large cohorts of ambulatory patients with HF, anemia was an independent risk factor for long-term mortality, regardless of whether patients had preserved or impaired LV systolic function. ${ }^{2,3}$ The aim of this study was to evaluate if the LVEF altered the prognostic value of anemia in patients admitted to an intensive care unit with decompensated chronic HF.

\section{Methods}

We consecutively selected patients admitted to the intensive care unit of a tertiary care hospital with decompensated HF from June 1, 2001, to December 1, 2003. Patients had to have $\geq 1$ hospitalization for symptomatic HF during the previous year. Patients were excluded if

\footnotetext{
${ }^{\mathrm{a}}$ Federal University of Bahia; and ${ }^{\mathrm{b}} \mathrm{Hospital}$ Aliança, Salvador, Brazil. Manuscript received March 24, 2006; revised manuscript received and accepted July 3, 2006.

*Corresponding author: Tel: 55-0-71-33344399; fax: 55-0-71-33344399.

E-mail address: adrianalatado@cardiol.br (A.L. Latado).
}

they had acute coronary syndromes as their main diagnoses. This study complied with the Declaration of Helsinki, ${ }^{4}$ and its protocol was approved by the ethics committee of our institution. All patients provided informed consent before inclusion.

Clinical and laboratory data were obtained directly from patients and their medical charts. The 12-lead electrocardiograms and laboratory results used for the analysis were obtained on admission. LVEFs were obtained from 2-dimensional echocardiograms and were available for 300 patients $(99 \%)$.

HF was defined on the basis of a history of worsening dyspnea or dyspnea at rest and signs of fluid retention or low cardiac output. ${ }^{5}$ After the evaluation of the on-call physician, 2 investigators reviewed the patients' clinical data to ascertain the diagnoses of HF before entering the study. If necessary, echocardiographic results were used to help the diagnosis of HF. Anemia was defined using the

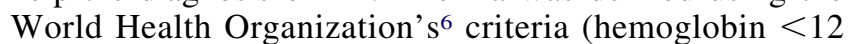
$\mathrm{g} / \mathrm{dl}$ in women and $<13 \mathrm{~g} / \mathrm{dl}$ in men). Renal dysfunction was defined as a creatinine level $>1.5 \mathrm{mg} / \mathrm{dl}$. Hyponatremia was considered present at a sodium level $<136$ $\mathrm{mEq} / \mathrm{L}$ and severe at a sodium level $<130 \mathrm{mEq} / \mathrm{L}$. LVEF was measured by echocardiography using Teicholz's or the modified Simpson's formula. HF with preserved LVEF was defined by a LVEF $\geq 45 \%$. Patients with LVEFs $<45 \%$ formed the group with systolic HF. 
Table 1

Baseline characteristics of patients according to left ventricular ejection fraction (LVEF)

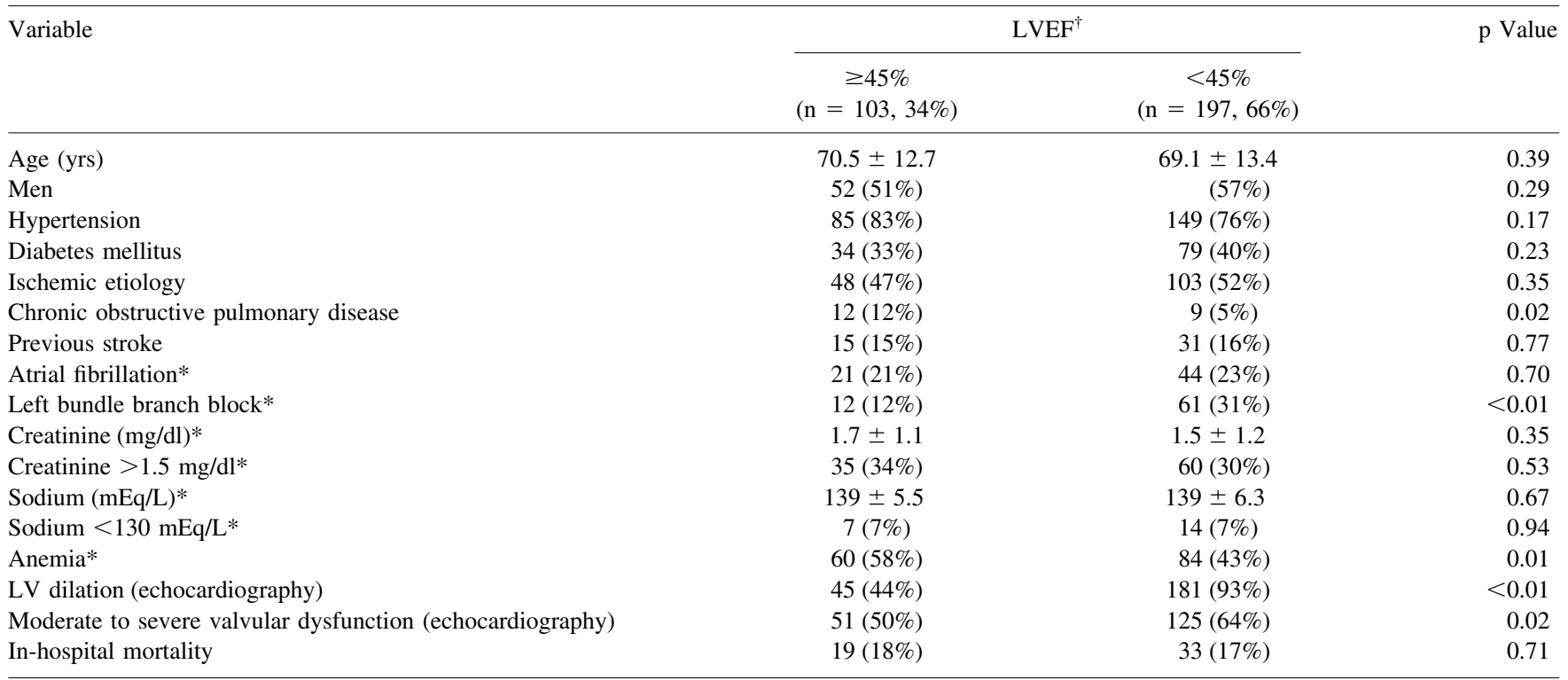

* Admission variables.

${ }^{\dagger}$ Only 300 patients (99\%) had information about LVEFs.

Quantitative variables were compared using the unpaired Student's $t$ test and are described as mean \pm SD. Categorical variables were compared using the chi-square test or Fisher's exact test and are described as proportions. Bivariate analysis was performed between anemic and nonanemic groups and between the groups with reduced and preserved LVEFs. Bivariate associations were described as relative risks (RRs) and their respective 95\% confidence intervals (CIs). Stratified analysis and the Breslow-Day homogeneity test (significance level 20\%) were used for the initial evaluation of interaction and confounding. Multivariate analysis with nonconditional logistic regression was used for the final evaluation of the interaction between anemia and LVEF on the mortality outcome. Logistic regression models (using a backward strategy) were also used in multivariate analysis for confounding of the association between anemia (the main independent variable) and inhospital mortality (the dependent variable). Variables were selected for confounding analysis if the $p$ values of their associations with anemia were $\leq 10 \%$ or by their clinical importance. The significance level for all other associations was 5\% (2 sided). SPSS version 10 for Windows (SPSS, Inc., Chicago, Illinois) was used for analysis.

\section{Results}

Three hundred three patients were evaluated. Their mean age was $69 \pm 13$ years, and $45.5 \%$ were women. The main cause of HF was coronary artery disease in half of the patients, followed by hypertension $(25.4 \%)$ and primary valvular heart disease $(11.6 \%)$. In the overall population, there were high prevalences of hypertension $(78 \%)$, diabetes mellitus (37.5\%), atrial fibrillation (21.5\%), and renal dysfunction on admission (32\%). HF with preserved LVEFs was present in 103 patients (34.3\%). Among patients with LV systolic dysfunction, the LVEF was $<30 \%$ in 132 pa- tients $(67 \%)$. Anemia on admission was also very common (47.5\%). In-hospital mortality (52 patients) was $17.3 \%$.

Table 1 lists the distribution of characteristics between the groups with preserved and reduced LVEFs. Anemia was significantly more frequent in patients with HF with preserved LVEFs than in patients with systolic dysfunction $(58.3 \%$ vs $42.6 \%, \mathrm{p}=0.01)$. There was no difference in in-hospital mortality between the groups with preserved and reduced LVEFs $(18.4 \%$ vs $16.8 \%, p=0.71)$.

Sample characteristics in the groups with and without anemia are listed in Table 2. Patients with anemia $(n=144)$ were older, had lower sodium levels, and had greater frequencies of renal dysfunction, ischemic causes of HF, and preserved LVEFs than patients without anemia.

Anemia on admission was significantly associated with a higher risk for death during hospitalization $(24.3 \%$ vs $10.7 \%$ in patients with and without anemia, respectively; RR 2.27, 95\% CI 1.33 to 3.88). After stratified analysis, a positive association between anemia and mortality occurred in the group with systolic dysfunction (RR 2.7) and the group with preserved LVEFs (RR 1.6) (Table 3). The results of the Breslow-Day homogeneity test were not significant $(\mathrm{p}=0.33)$.

Clinical variables included in the multivariate models for confounding analysis were gender, age, atrial fibrillation, ischemic cause of HF, diabetes mellitus, renal dysfunction, hyponatremia, and LV systolic function. After adjustment, anemia remained independently associated with in-hospital mortality (odds ratio 2.7, 95\% CI 1.43 to $5.04, \mathrm{p}=0.002$; Table 3). An interaction term was included in the multivariate model, and no significant interaction was verified between anemia and LV systolic function relative to the mortality outcome ( $\mathrm{p}=0.08$ for interaction). We performed additional analysis (bivariate and multivariate) excluding patients with $\mathrm{HF}$ due to valvular disease $(11.6 \%)$, and the 
Table 2

Baseline characteristics of patients with heart failure with and without anemia

\begin{tabular}{|c|c|c|c|}
\hline Variable & \multicolumn{2}{|c|}{ Anemia } & $\mathrm{p}$ Value \\
\hline Women & $73(51 \%)$ & $65(41 \%)$ & 0.09 \\
\hline Hypertension & $114(79 \%)$ & $122(77 \%)$ & 0.61 \\
\hline Diabetes mellitus & $60(42 \%)$ & $54(34 \%)$ & 0.17 \\
\hline Previous stroke & $25(18 \%)$ & $21(13 \%)$ & 0.30 \\
\hline Atrial fibrillation* & $37(26 \%)$ & $28(18 \%)$ & 0.10 \\
\hline Creatinine $(\mathrm{mg} / \mathrm{dl})^{*}$ & $1.8 \pm 3.7$ & $1.5 \pm 0.58$ & $\leq 0.01$ \\
\hline Creatinine $>1.5 \mathrm{mg} / \mathrm{dl}^{*}$ & $58(40 \%)$ & $38(24 \%)$ & 0.002 \\
\hline LV dilatation (echocardiography) & $104(73 \%)$ & $123(80 \%)$ & 0.15 \\
\hline LVEF $\geq 45 \%$ (echocardiography) & $60(42 \%)$ & $43(28 \%)$ & 0.01 \\
\hline
\end{tabular}

* Admission variables.

Table 3

Association between anemia and in-hospital mortality

\begin{tabular}{lcccc}
\hline Variable & \multicolumn{2}{c}{ In-Hospital Mortality Anemia } & Risk Ratio & 95\% CI \\
\cline { 2 - 4 } & Yes & No & & \\
\hline All patients & $35(24.3 \%)$ & $17(10.9 \%)$ & $2.27^{*}$ & $1.33-3.88$ \\
& & & $2.68^{\dagger}$ & $1.43-5.04$ \\
LVEF $\geq 45 \%$ & $13(21.7 \%)$ & $6(14.0 \%)$ & $1.55^{*}$ & $0.04-3.76$ \\
LVEF $<45 \%$ & $22(26.2 \%)$ & $11(9.7 \%)$ & $2.69^{*}$ & 0.002 \\
Breslow-Day & & & 0.32 & 0.002 \\
Interaction test $^{\ddagger}$ & & & & 0.33 \\
\hline
\end{tabular}

* RR; ${ }^{\dagger}$ odds ratio, obtained by logistic regression.

Homogeneity test; ${ }^{\S}$ chi-square test for interaction between anemia and LVEF.

results were similar (odds ratio $2.4,95 \%$ CI 1.1 to $5.0, \mathrm{p}=$ 0.14 for interaction between anemia and LVEF).

\section{Discussion}

The present study demonstrated that anemia was a frequent finding in patients hospitalized for severe decompensated HF $(47.5 \%)$ and even more prevalent in those with preserved LVEFs (58.3\%). Additionally, it was shown, for the first time in this specific population, that anemia was an independent predictor of in-hospital mortality, regardless of whether patients had preserved or impaired LV systolic function.

Previous studies have already demonstrated a high prevalence of anemia in patients with chronic HF, as well as its independent association with worse long-term survival. ${ }^{7-12}$ However, the population used in these studies was composed of ambulatory patients with HF and systolic LV dysfunction (LVEF $<40 \%$ ), systematically excluding patients with preserved LVEFs, a group that represents up to $50 \%$ of the overall HF population. ${ }^{13}$ In our study, patients with preserved LVEFs constituted $34 \%$ of the overall population. Therefore, it is necessary to investigate if predictors of outcomes in systolic HF also have a prognostic impact on HF with a preserved LVEF.
Few studies have examined the association between anemia and adverse outcomes in patients with HF and preserved LVEFs. Brucks et $\mathrm{al}^{1}$ demonstrated a higher readmission rate in patients with anemia but did not find a significant difference in late survival of this group in comparison with patients without anemia. That study was composed of outpatients with HF and LVEFs $>50 \%$ but also had several exclusion criteria, such as active myocardial ischemia, myocardial infarction, dialysis-dependent renal failure, malignancy, hemodynamically significant valve disease, and prosthetic valve replacement.

More recently, 2 other studies have evaluated the prognostic role of anemia in large cohorts of patients with HF, including a significant proportion of patients with preserved LVEFs. ${ }^{2,3}$ Felker et $\mathrm{al}^{2}$ evaluated a large sample of patients with symptomatic HF who were referred to diagnostic cardiac catheterization. Of 4,951 patients, 3,093 had preserved LVEFs $(>40 \%)$. Their results showed that anemia was an independent risk factor for mortality in groups with preserved and impaired systolic function, and there was no interaction between anemia and the LVEF. O'Meara et $\mathrm{al}^{3}$ published a substudy of the Candesartan in Heart Failure: Assessment of Reduction in Mortality and Morbidity (CHARM) trial with 2,653 patients with HF, of whom 1,069 
had preserved EF. Once again, anemia was shown to be an independent risk factor for mortality, irrespective of LVEF. The investigators also showed an interesting inverse correlation between hemoglobin and LVEF, a finding that is consistent with our observation of a higher prevalence of anemia in the group with preserved LVEFs.

The currently available data on the prognostic role of anemia in HF with a preserved LVEF are not conclusive. All described studies had methodologic issues that may in part limit the generalization of their results to a larger and less selected HF population. In the smallest study, ${ }^{1}$ patients were young and ambulatory and were excluded if they had important co-morbidities associated with HF. Although Felker et $\mathrm{al}^{2}$ evaluated the largest cohort of patients with HF and preserved LVEFs, their study was a retrospective analysis of a clinical database comprising patients who underwent cardiac catheterization and was therefore subject to the limitations of such studies. In the CHARM substudy, ${ }^{3}$ the greatest limitation was related to the population, which was derived from a clinical trial, with its typically restrictive inclusion and exclusion criteria.

Unlike the previously mentioned studies, the current study prospectively enrolled consecutive patients with severe decompensated HF, excluding only those with concomitant diagnoses of acute coronary syndromes. Thus, our sample was representative of a large HF population hospitalized for worsening symptoms, which in the United States constitutes 1,000,000 patients every year. ${ }^{14}$ Additionally, in contrast to other reports, our study evaluated in-hospital mortality, a short-term outcome.

The present study had some limitations. Our sample was relatively small, which could have interfered with the results of more complex statistical analysis. Our results should not be generalized to the overall HF population. We enrolled patients with severe decompensated HF, so the results of this study should be applied only to this specific population. Our patients had a high frequency of moderate to severe valvular dysfunction, independent of LVEF. Most of them had mitral regurgitation, probably because of LV dilation, because primary valvular heart disease was the main cause of HF in only $11.6 \%$. However, because the diagnosis of HF with a preserved LVEF did not rely on the documentation of severe diastolic abnormalities, it is possible that the valvular dysfunction could have been responsible for the HF syndrome in some patients with preserved LVEFs. In contrast, our risk estimates did not change after the exclusion of patients with $\mathrm{HF}$ and chronic valvular cardiomyopathy. We did not have detailed information on medical treatments or levels of recent biomarkers, which could have prognostic importance.

1. Brucks S, Little WC, Chao T, Rideman RL, Upadhya B, WesleyFarrington D, Sane DC. Relation of anemia to diastolic heart failure and the effect on outcome. Am J Cardiol 2004;93:1055-1057.

2. Felker GM, Shaw LK, Stough WG, O'Connor CM. Anemia in patients with heart failure and preserved systolic function. Am Heart J 2006; 151:457-462.

3. O'Meara E, Clayton T, McEntegart MB, McMurray JJ, Lang CC, Roger SD, Young JB, Solomon SD, Granger CB, Ostergren J, et al. CHARM Committees and Investigators. Clinical correlates and consequences of anemia in a broad spectrum of patients with heart failure. Results of the Candesartan in Heart Failure: Assessment of Reduction in Mortality and Morbidity (CHARM) program. Circulation 2006;113: 986-994.

4. World Medical Organization. Declaration of Helsinki. BMJ 1996;313: 1448-1449.

5. Task Force on Heart Failure of the European Society of Cardiology. Guidelines for the diagnosis of heart failure. Eur Heart $J$ 1995;16: 741-751.

6. World Health Organization. Nutritional anaemias: report of a WHO scientific group. WHO Tech Rep Ser 1968;405:3-37.

7. Al-Ahmad A, Rand WN, Manjunath G, Konstam MA, Salem DN, Levey AS, Sarnak MJ. Reduced kidney function and anemia as risk factors for mortality in patients with left ventricular dysfunction. $J \mathrm{Am}$ Coll Cardiol 2001;38:955-962.

8. Horwich TB, Fonarow GC, Hamilton MA, MacLellan WR, Borenstein $\mathrm{J}$. Anemia is associated with worse symptoms, greater impairment in functional capacity and a significant increase in mortality in patients with advanced heart failure. J Am Coll Cardiol 2002;39:1780-1786.

9. Mozaffarian D, Nye R, Levy WC. Anemia predicts mortality in severe heart failure. J Am Coll Cardiol 2003;41:1933-1939.

10. Ezekowitz JA, McAlister FA, Armstrong PW. Anemia is common in heart failure and is associated with poor outcomes. Circulation 2003; 107:223-225.

11. Anand I, McMurray JJV, Whitmore J, Warren M, Pham A, McCamish MA, Burton PB. Anemia and its relationship to clinical outcome in heart failure. Circulation 2004;110:149-154.

12. Maggioni AP, Opasich C, Anand I, Barlera S, Carbonieri E, Gonzini L, Tavazzi L, Latini R, Cohn J. Anemia in patients with heart failure: prevalence and prognostic role in a controlled trial and in clinical practice. J Card Fail 2005;11:91-98.

13. Zile MR. Heart failure with preserved ejection fraction: is this diastolic heart failure? J Am Coll Cardiol 2003;41:1519-1522.

14. Thom T, Haase N, Rosamond W, Howard VJ, Rumsfeld J, Manolio T, Zheng ZJ, Flegal K, O'Donnell C, Kittner S, et al. American Heart Association Statistics Committee and Stroke Statistics Subcommittee. Heart disease and stroke statistics-2006 update: a report from the American Heart Association Statistics Committee and Stroke Statistics Subcommittee. Circulation 2006;113:e85-e151. 